\title{
Rasgos tomográficos de los septos del seno maxilar en pacientes de la clínica dental docente de la Universidad Peruana Cayetano Heredia
}

Tomographic features of the maxillary sinus septa in patients of the dental clinic of the Universidad Peruana Cayetano Heredia.

Rosa Elizabeth Cruz Sánchez ${ }^{1,2, a, b}$, Alexis Evangelista Alva 2,a,c, Milushka Miroslava Quezada Márquez 2,a,b,c

\section{RESUMEN}

Los septos del seno maxilar son variantes anatómicas que se forman como resultado de los residuos de la hipoplasia en diferentes áreas del proceso alveolar siendo de forma, tamaño y número variable. Su hallazgo se puede realizar mediante radiografías panorámicas de rutina. La importancia de un septo en el seno maxilar es que puede generar modificaciones para las cirugías, pudiendo ser necesario abrir dos o más ventanas quirúrgicas para su remoción.Objetivo: La finalidad de este estudio fue analizar los rasgos tomográficos de los septos del seno maxilar en pacientes que asistieron entre los años 2014 - 2016 a la clínica dental docente de la Universidad Peruana Cayetano Heredia. Materiales y métodos: Esta investigación fue de tipo transversal y descriptivo, para ello se examinaron 813 tomografías computarizadas volumétricas. La prueba de chi cuadrado y la estadística descriptiva se usaron para el análisis, con un intervalo de confianza al $95 \%$, con un valor $\mathrm{p}<0.05$. Resultados: 298 pacientes presentaron septos, presentando mayor porcentaje los septos congénitos $(69.2 \%)$ y fueron más comunes en pacientes de sexo femenino con un $36 \%$. Conclusiones: prevalecieron los septos de tipo congénito, lo que indica que se formaron a partir del desarrollo radicular.

PALABRAS CLAVE: Tomografía computarizada volumétrica, seno maxilar, variación anatómica. 


\section{SUMMARY}

The septa of the maxillary sinus are anatomical variants that are formed as a result of the residues of hypoplasia in different areas of the alveolar process, being of variable shape, size and number. Your finding can be made using routine panoramic radiographs. The importance of a septum in the maxillary sinus is that it can generate modifications for maxillary sinus surgeries, and it may be necessary to open two or more surgical windows for its removal. Objective: The purpose of this study was to analyze the tomographic features of the maxillary sinus septa in patients who attended the teaching dental clinic of the Universidad Peruana Cayetano Heredia between 2014- 2016. Materials and methods: This research was cross-sectional and descriptive, for which 813 cone beam CT scans were examined. Chi square test and descriptive statistics were used for the analysis, with a 95\% confidence interval, with a value $\mathrm{p}<0.05$. Results: 298 patients presented septa, with a higher percentage of congenital septa (69.2\%) and were more common in female patients with $36 \%$. Conclusions: the congenital type septa prevailed, which indicates that they were formed from root development.

\section{KEY WORDS: Volumetric computed tomography, maxillary sinus, anatomical variation.}

\section{INTRODUCCIÓN}

Con la evolución de la radiología, desde las imágenes bidimensionales a las tridimensionales, se pueden visualizar con mayor detalle estructuras anatómicas y variantes anatómicas del macizo facial, lo que ha logrado un gran valor para el éxito, diagnóstico quirúrgico y postquirúrgico sobre todo en los campos de cirugía buco maxilofacial, implantología y periodoncia.

La importancia de evaluar el seno maxilar con tomografía computarizada volumétrica (TCV) es que se elimina la superposición con otras estructuras anatómicas $(1,2,3)$, por lo tanto se puede obtener mejor información de la estructura interna de ésta cavidad y así mismo los septos pueden ser identificados con mayor facilidad $(4,5)$.

Los septos suelen ser crestas óseas puntiagudas o con forma de arco gótico $(6,7,8,9)$ que se ubican en el seno maxilar, generalmente son hallazgos radiológicos, son de tamaño variable llegando incluso a dividir el seno completamente en dos o más cavidades (1, 2,10-13), su diagnóstico diferencial son las exostosis, sin embargo éstas tienen forma redondeada $(6,7,8)$ (figura 1$)$.

En 1990 Underwood fue el primero en describir los septos sensuales y mencionó que su origen más frecuente es en la pared lateral o inferior del seno maxilar (14).

Algunos autores relacionan la aparición de septos con

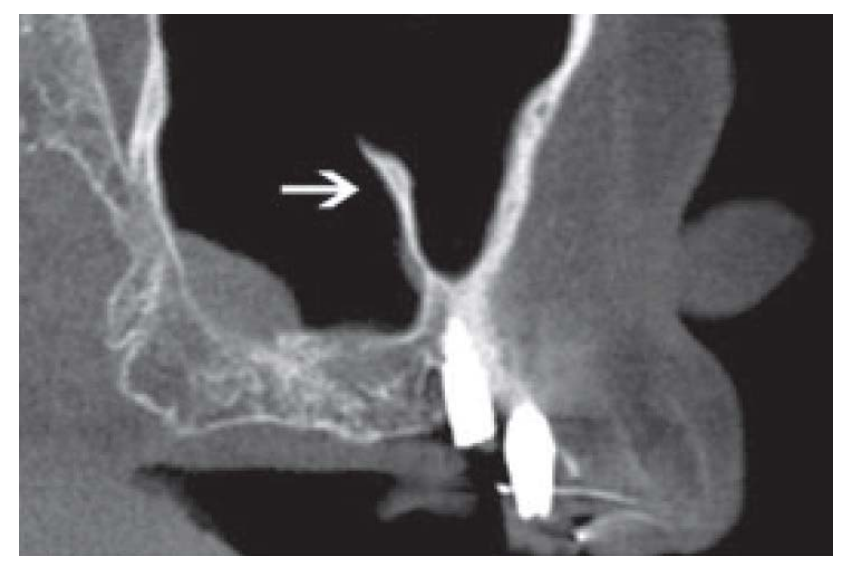

Figura 1. Septo sinusal (imagen tomada de Pelinsari et al. ${ }^{8}$ ).

la pérdida de piezas dentarias, ya que se producen cambios morfológicos y funcionales a nivel extraoral e intraoral $(1,2,10,11,12,13)$ otros refieren su origen a los residuos de la hipoplasia en diferentes zonas del proceso alveolar $(6,10,15)$.

De acuerdo a su origen, existen dos tipos de septos: los primarios y los secundarios. Los primarios o llamados también septos congénitos, se forman durante el desarrollo de la zona central de la cara, originándose de la fusión de residuos durante la formación del seno maxilar (12), (figura 2 y 3); sin embargo Underwood relaciona su origen con las piezas dentarias justificando que se forman por separaciones intermedias del piso antral debido al desarrollo radicular (14). Autores como Lee et al., mencionan que los septos se originan por proyecciones digitales debido a la invaginación del infundíbulo etmoidal durante el desarrollo 


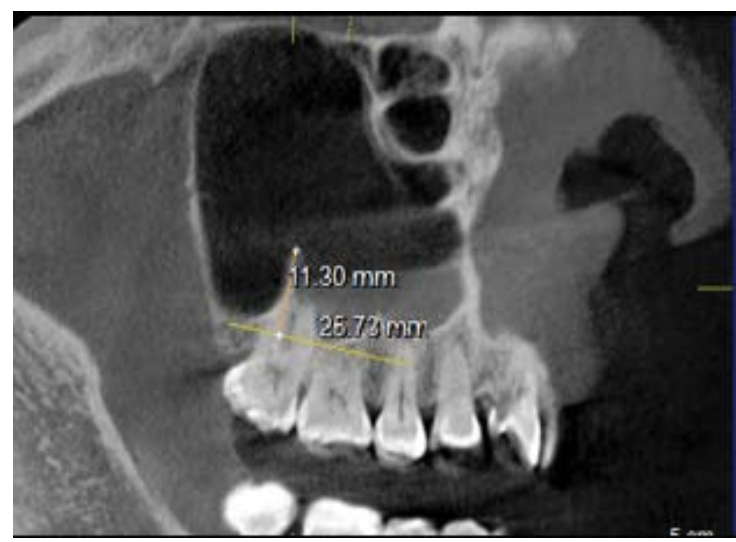

Figura 2. Medida de septo congénito (presencia de septo adyacente a pieza dentaria). (Imagen tomada de uno de los pacientes de este estudio).

embrionario donde las paredes adyacentes no se reabsorben (2).

Los septos secundarios, tienen un origen adquirido asociado a atrofia del hueso maxilar como consecuencia
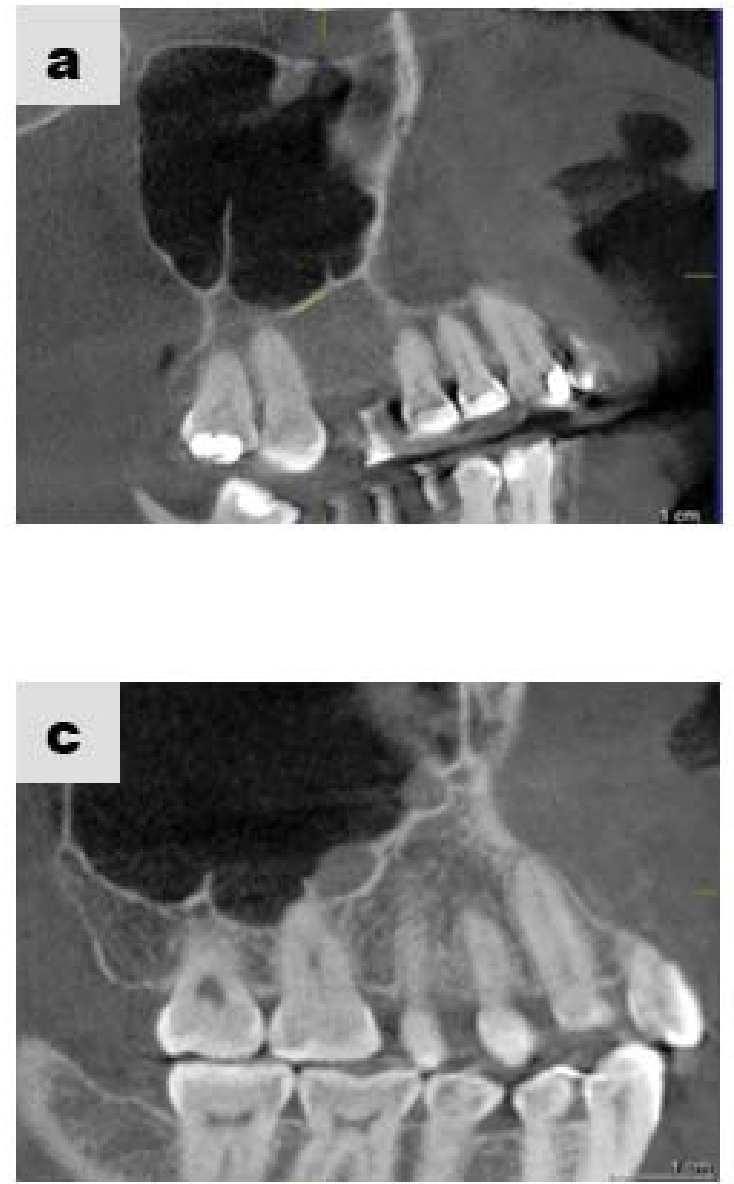

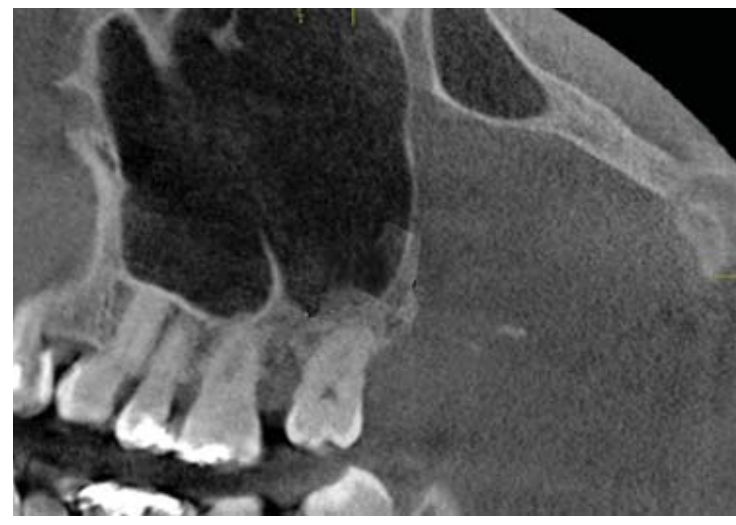

Figura 3. Otros septos. (Imagen tomada de uno de los pacientes de este estudio).

de pérdida de dientes, siendo más frecuentes en el piso del seno. La longitud de éstos septos suele ser inferior que los primarios $(2,7,12,15,16,17,18)$. (figura 4 y 5 ). Gonzales et al., consideraron septos primarios a aquellos donde hay piezas dentarias presentes y los septos secundarios en zonas edéntulas (6).
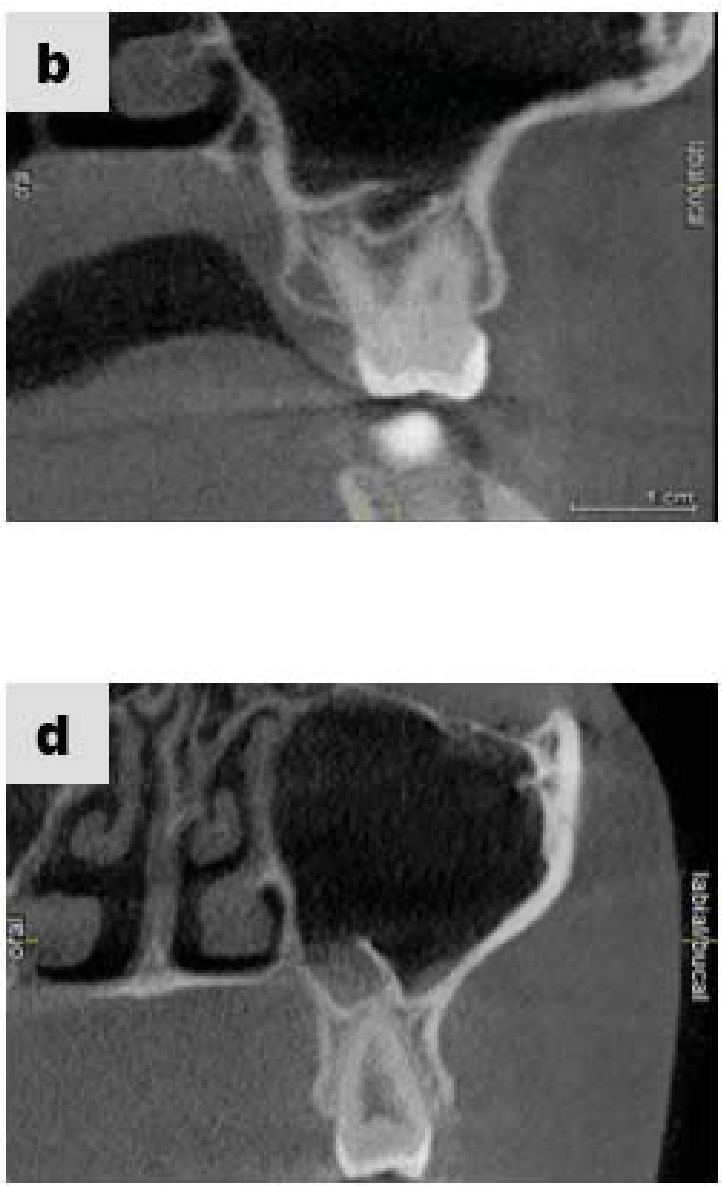

Figura 4. Septos congénitos (a,b,c,d). (Imagen tomada de uno de los pacientes de este estudio). 


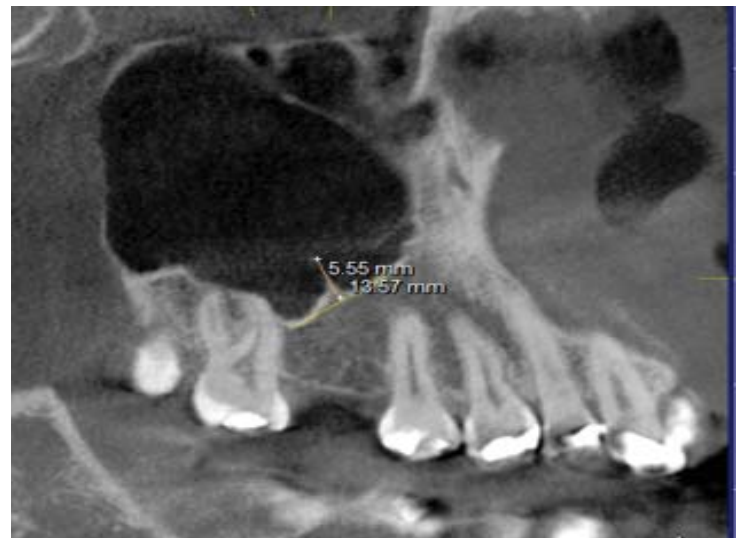

Figura 5. Medida de otros septos (presencia de septo adyacente a zona edéntula). (Imagen tomada de uno de los pacientes de este estudio).

En este trabajo de investigación se evaluaron 458 septos, mediante TCV considerando pacientes de ambos sexos, con edades de 18 años a más, las características que se consideraron fueron: tipo de septo (congénito u otros septos), localización (bilateralidad y lado), longitud (es la medida del septo desde la unión de los lóbulos del seno maxilar hasta su porción más distal). La finalidad de este reporte fue revisar los rasgos tomográficos de los septos en el seno maxilar para que no pasen desapercibidos por los cirujanos maxilofaciales, implantólogos y periodoncistas, ya que la presencia de uno o más septos implica realizar cambios en la angulación de los implantes dentarios (1$3,5,7,12)$, así como la creación de más de una ventana quirúrgica en los procedimientos de elevación de seno maxilar, para evitar la perforación de la membrana de Schneider $(13,16,17,19,20)$.

\section{MATERIAL Y MÉTODOS}

El diseño de este estudio fue de tipo descriptivo y retrospectivo, utilizando TCV que cumplan con los criterios de selección adquiridas del servicio de radiología oral y maxilofacial del Posgrado de la Facultad de Estomatología de la UPCH, dentro del periodo 2015 al 2016. Se incluyeron TCV de pacientes mayores de 18 años de edad, con presencia de septos, pacientes de ambos sexos y TCV nítidas que permitan la visualización de ambos senos maxilares; se excluyeron TCV de pacientes con tratamientos quirúrgicos que comprometían los senos maxilares, pacientes con patologías quísticas o tumorales en el maxilar superior, pacientes con fracturas y con malformaciones cráneo-faciales.
La investigadora principal fue calibrada y capacitada por un especialista en radiología oral y maxilofacial con más de 10 años de experiencia. La calibración se llevó a cabo en 23 TCV considerando la variable localización (zona, bilateralidad y lado) con un equipo tomográfico GALILEOS® (Sirona Dental Systems Gmbh FabrikstraBe31, Bensheim, Alemania) que opera de $10 \mathrm{~mA}$ a $42 \mathrm{~mA}$ y $85 \mathrm{Kv}$ de acuerdo a cada paciente, las imágenes fueron exportadas a una computadora Lenovo H61 y evaluadas a través del software Galileos 1.7.2.

Se utilizó la prueba Kappa para evaluar el tipo de septo, obteniéndose un valor de 0,92 (intraobservador) y 0,87 (interobservador). Asimismo se evaluó la longitud de los septos a través del coeficiente de correlación intraclase obteniéndose 0,89 (intraobservador) y 0,85 (interobservador). Para la comparación de la variable septo del seno maxilar: según sexo, edad, tipo, localización, longitud y número se utilizaron pruebas de Chi Cuadrado y T de Student. El estudio contó con un nivel de confianza de $95 \%$ y un nivel de significancia de 0,05 . Los análisis fueron realizados en el paquete estadístico SPSS V.22.0 para Windows.

La visualización de TCV por sesiones fue en horas de la mañana realizando un descanso visual cada 15 minutos por cada 2 horas de trabajo en el computador y cada 20 minutos realizar una pausa y fijar la mirada en un punto lejano (21).

Las imágenes digitales se analizaron en una pantalla de alta resolución de 21 pulgadas, fijada en 1600x900 píxeles con una profundidad de color de 32 bits. Las variables y los datos se registraron en una ficha de recolección (en el programa de Excel-Microsoft Office.).

\section{RESULTADOS}

Se analizaron 813 TCV de los cuales el 36,7\% (298 pacientes) presentaron septos en el seno maxilar, siendo $36 \%$ pacientes de sexo femenino y $37,9 \%$ de sexo masculino (el motivo por el cual los hombres presentan mayor porcentaje que las mujeres es porque existe menor cantidad de varones en el estudio); sin embargo debido a que hubo mayor afluencia de pacientes mujeres, ellas presentaron mayor cantidad de septos.

El rango de edades de los pacientes de este estudio fue desde los 18 hasta los 85,9 años, siendo la edad 
media de 46,7 años, con una desviación estándar de 14,5 años. La edad promedio de las mujeres fue de 46,7 años y de los hombres fue de 46,6 años (tabla 1). De acuerdo al tipo de septos, considerando la clasificación de Shen et al., se encontraron 317 (69,2\%) septos congénitos y $141(30,8 \%)$ otros septos (19) (tabla 2).

Se encontraron $298(65,1 \%)$ septos unilaterales y
$160(34,9 \%)$ bilaterales (tabla 3); considerando lado, la frecuencia de septos predominó en el lado derecho con 234 y el lado izquierdo presentó un total de 224 septos (tabla 4). La longitud promedio de septos congénitos fue de $5,8 \mathrm{~mm}$ y para otros septos fue de $5.6 \mathrm{~mm}$. El valor p fue igual a 0,523 lo que indica que no hay diferencias estadísticamente significativas entre ambos grupos (tabla 5).

Tabla 1. Distribución de la edad y sexo de los pacientes que presentaron septos evaluados con TCV

\begin{tabular}{lcccccc}
\hline \multicolumn{1}{c}{ SEXO } & TOTAL & $\mathbf{n}$ & MEDIA (AÑOS) & $\begin{array}{c}\text { DESVIACIÓN } \\
\text { ESTÁNDAR }\end{array}$ & \multicolumn{2}{c}{ EDAD } \\
& & & & & MÍNIMA & MÁXIMA \\
\hline MASCULINO & 261 & $99(37.9 \%)$ & 46,6 & 16.0 & 18,9 & 81,2 \\
FEMENINO & 552 & $199(36 \%)$ & 46,7 & 13.7 & 18,0 & 85,9 \\
TOTAL & 813 & $298(36.7)$ & 46,7 & 14.5 & 18,0 & 85,9 \\
\hline
\end{tabular}

$p=0,604$ Comparación de proporciones.

Tabla 2. Distribución de septos según tipo*, de los pacientes evaluados con TCV.

\begin{tabular}{lll}
\hline TIPO & n & $\%$ \\
\hline CONGÉNITO & 317 & 69,2 \\
OTROS SEPTOS & 141 & 30,8 \\
TOTAL & 458 & 100,0 \\
\hline * Clasificación de She et al.,(20) & & \\
n= Frecuencia absoluta & \\
\%= Frecuencia relativa. &
\end{tabular}

Tabla 3. Distribución de frecuencia de septos según localización (bilateralidad).

\begin{tabular}{lll}
\hline BILATERALIDAD & n & \% \\
\hline UNILATERAL & 298 & 65,1 \\
BILATERAL & 160 & 34,9 \\
TOTAL & 458 & 100,0 \\
\hline n= Frecuencia absoluta & & \\
$\%=$ Frecuencia relativa. & &
\end{tabular}


Tabla 4. Distribución de septos según número y lado evaluados con TCV

\begin{tabular}{lllllll}
\hline \multirow{2}{*}{ NÚMERO } & \multicolumn{2}{c}{ LADO } & & & \multicolumn{2}{c}{ TOTAL } \\
& DERECHO & & IZQUIERDO & n & \% \\
& $\mathbf{n}$ & $\mathbf{0}$ & $\mathbf{n}$ & $\mathbf{\%}$ & & \\
$\mathbf{1}$ & 173 & 49,3 & 178 & 50,7 & 351 & 100 \\
$\mathbf{2}$ & 49 & 63,6 & 28 & 36,4 & 77 & 100 \\
$\mathbf{3}$ & 8 & 40,8 & 18 & 69,2 & 26 & 100 \\
$\mathbf{4}$ & 4 & 100 & 0 & 0 & 4 & 100 \\
TOTAL & 234 & & & & 458 & \\
\hline
\end{tabular}

$\mathrm{n}=$ Frecuencia absoluta

$\%=$ Frecuencia relativa

Tabla 5. Distribución de longitud de septos según tipo*, de pacientes evaluados con TCV.

\begin{tabular}{llllll}
\hline \multirow{2}{*}{ TIPO DE SEPTO } & \multicolumn{1}{c}{ MEDIA } & MÍNIMA & MÁXIMA & DESVIACIÓN ESTÁNDAR & n \\
& $(\mathrm{mm})$ & $(\mathrm{mm})$ & $(\mathrm{mm})$ & & 317 \\
\hline CONGÉNITO & 5,8 & 1,7 & 28,9 & 3,4 & 141 \\
OTROS SEPTOS & 5,6 & 1,6 & 15,7 & 3,2 & 458 \\
TOTAL & & & & & \\
\hline
\end{tabular}

*Clasificación de She et al., (20)

$\mathrm{n}=$ Frecuencia absoluta.

$\mathrm{p}=0.523$.

Tabla 6. Tabla resumen de prevalencia de septos según sexo de otros estudios con TCV.

\begin{tabular}{|c|c|c|c|c|c|c|c|c|}
\hline \multirow[b]{2}{*}{ AUTOR } & \multirow[b]{2}{*}{ AÑNO } & \multirow[b]{2}{*}{$\begin{array}{l}\text { TIPO DE } \\
\text { EXAMEN }\end{array}$} & \multicolumn{3}{|c|}{ POBLACIÓN } & \multicolumn{3}{|c|}{ PACIENTES CON SEPTOS } \\
\hline & & & $\mathbf{M}$ & $\mathbf{F}$ & TOTAL & $\mathbf{M}$ & $\mathbf{F}$ & TOTAL \\
\hline Cruz (24) & 2017 & $\mathrm{TCHC}$ & 261 & 552 & 813 & 99 & 199 & 298 \\
\hline Neugebauer et al.,(21) & 2010 & $\mathrm{TCHC}$ & $\ldots$ & $\ldots$ & 1029 & $\ldots$ & $\ldots$ & 484 \\
\hline Lana et al.(8) & 2012 & TCHC & 238 & 262 & 500 & $\ldots$ & $\ldots$ & 222 \\
\hline Özec et al.(12) & 2008 & $\mathrm{TCHC}$ & $\ldots$ & $\ldots$ & 575 & $\ldots$ & $\ldots$ & $13,7 \%$ \\
\hline Shen et al.(20) & 2012 & $\mathrm{TC}$ & 207 & 216 & 423 & 72 & 52 & 124 \\
\hline Dobele et al.(4) & 2013 & $\mathrm{TCHC}$ & 18 & 16 & 34 & $\ldots$ & $\ldots$ & 7 \\
\hline Quian et al.(22) & 2016 & $\mathrm{TCHC}$ & 168 & 338 & 506 & 76 & 83 & 159 \\
\hline Orhan et al.(11) & 2013 & $\mathrm{TCHC}$ & 120 & 152 & 272 & $50 \%$ & $44 \%$ & 228 \\
\hline Van Zyl et al.(18) & 2009 & $\mathrm{TCHC}$ & 88 & 112 & 200 & $\ldots$ & $\ldots$ & 138 \\
\hline Rancitelli et al.(23) & 2015 & $\mathrm{TCHC}$ & 42 & 72 & 114 & 27 & 36 & 63 \\
\hline
\end{tabular}




\section{DISCUSIÓN}

En este artículo se evaluaron 298 pacientes con esta variante anatómica, con la finalidad de valorar mediante TCV la ubicación, longitud, forma, tipo, frecuencia, entre otras, de cada uno de los septos intrasinusales.

Se encontraron 12 estudios de revisión que evaluaron los septos con la misma herramienta imagenológica (tomografía computarizada volumétrica).

En este estudio el $36.7 \%$ de pacientes presentaron septos intrasinusales, considerando una altura mínima de $1.6 \mathrm{~mm}$, coincidiendo con estudios de Dobele et al., Lana et al., Özec et al., Shen et al., Neugebauer et al., y Quian et al., donde obtuvieron valores comprendidos entre $13,7 \%-47 \%$, con las misma medidas mínimas de los septos $(4,8,12,19,22,23)$ (tabla 6).

Pacientes de sexo femenino tuvieron mayor prevalencia de septos, coincidiendo con estudios realizados por Rancitelli et al., y Quian et al., (20,23). La razón de estos resultados se puede asociar a la afluencia de pacientes de este sexo en la recolección de datos.

En este reporte se consideró septos congénitos llamados también septos primarios que estuvieron en relación con la presencia de piezas dentarias y otros septos donde hubo ausencia de dientes, se empleó este segundo término considerando la justificación de Shen et al., (19), ya que al no existir evidencias imagenológicas previo a la exodoncia no se puede confirmar si el septo estuvo presente antes de la cirugía o si se formó después. De estos dos tipos, los septos congénitos tuvieron mayor porcentaje $(69,2 \%)$. Otros estudios como los de Rancitelli et al., encontraron mayor prevalencia de septos primario con un total de 60 septos (20); Orhan et al., clasificaron los septos en primarios y otros septos, mostrando resultados similares a este estudio (11) (tabla 7).

Rosano et al., Maestre et al., y Van Zyl et al., indicaron que los septos primarios se forman debido al desarrollo radicular, producto de ello habrán separaciones intermedias del piso antral, dando lugar a la localización de los tabiques entre raíces dentarias, este concepto justificaría su predominio en esta población $(7,13,18)$.

El promedio total fue de 458 septos, la mayoría de éstos (con un total de 298) se presentaron en un solo seno, coincidiendo con estudios realizados por Lana et al., y Rancitelli et al., revelando que la formación de un septo es independiente del seno maxilar $(8,22)$. Así mismo estudios realizados por Shahidi et al., Orhan et al., y Özec et al., encontraron mayor cantidad de septos bilaterales $(5,11,12)$, pudiendo coincidir con Ella et al., quienes mencionaron que existe una prevalencia del $70 \%$ que los pacientes presenten la misma configuración de septos en ambos senos maxilares (10) (tabla 8).

En este estudio hubo un paciente que presentó cuatro de septos en un seno, siendo dos congénitos y dos otros septos, coincidiendo con Van Zyl et al., quienes mencionan en su estudio esta cantidad máxima de septos (18). La presencia de múltiples septos está asociada a la atrofia que sufre el maxilar después de una exodoncia.

Tabla 7. Tabla resumen de otro estudio con TCV donde consideraron tipos de septos.

\begin{tabular}{lcccl}
\hline AUTOR & AÑo & $\begin{array}{c}\text { TIPO DE } \\
\text { EXAMEN }\end{array}$ & $\begin{array}{c}\mathbf{n}^{\circ} \text { DE } \\
\text { SEPTOS }\end{array}$ & TIPOS DE SEPTOS \\
\hline Cruz (24) & 2017 & TCHC & 458 & $\begin{array}{l}\text { CONGÉNITOS }=317(69,2 \%) \\
\text { OTROS SEPTOS }=141(30,84 \%)\end{array}$ \\
& & & & \\
Rancitelli et al.(23) & 2015 & TCHC & 95 & $\begin{array}{l}\text { PRIMARIOS O CONGÉTITOS }=60 \\
\text { SECUNDARIOS U OTROS SEPTOS }=35\end{array}$ \\
& & & &
\end{tabular}


Tabla 8. Tabla resumen de estudios donde consideraron septos de acuerdo a bilateralidad.

\begin{tabular}{lcccc}
\hline & & & \multicolumn{2}{c}{ BILATERALIDAD } \\
AUTOR & AÑ & $\begin{array}{c}\text { TIPO DE } \\
\text { EXAMEN }\end{array}$ & UNILATERAL & BILATERAL \\
\hline Cruz (24) & 2017 & TCHC & 298 & 160 \\
Lana et al.(8) & 2012 & TCHC & 121 & 101 \\
Rancitelli et al.(23) & 2015 & TCHC & 39 & 24 \\
Orhan et al.(11) & 2013 & TCHC & 148 & 211 \\
Özec et al.(12) & 2008 & TCHC & $2,1 \%$ & $6,4 \%$ \\
Shahidi et al., (5) & 2016 & TCHC & 70 & 110 \\
\hline
\end{tabular}

\section{CONCLUSIONES}

Dentro de los rasgos tomográficos de los septos del seno maxilar, resaltaron: el tipo de septo primario y el valor máximo de septos que se encontraron en un solo seno maxilar fue de cuatro. Los septos más frecuentes se observaron en pacientes del sexo femenino, septos congénitos y unilaterales en el lado derecho. Por lo tanto estos rasgos tomográficos deben ser evaluados por los odontólogos, principalmente por los cirujanos maxilofaciales, implantólogos y peridoncistas.

\section{Correspondencia:}

Rosa Elizabeth Cruz Sánchez

Correo electrónico: rosa.cruz.s@upch.pe

\section{REFERENCIAS BIBLIOGRAFICAS}

1. 1. Betts N, Miloro M. Modification of the sinus lift procedure for septa in the maxillary antrum. J Oral Maxillofac Surg. 1994;52(3):332-3.

2. 2. Lee WJ, Lee SJ, Kim HS. Analysis of location and prevalence of maxillary sinus septa. J Periodontal Implant Sci. 2010;40(2):56-60.

3. 3. Pommer B, Ulm C, Lorenzoni M, Palmer R, Watzek G, Zechner W. Prevalence, location and morphology of maxillary sinus septa: systematic review and meta-analysis. J Clin Periodontol. 2012;39(8):769-73.

4. 4. Dobele I, Kise L, Apse P, Kragis G, Bigestans A. Radiographic assessment of findings in the maxillary sinus using cone-beam computed tomography. Stomatologija. 2013;15(4):119-22.

5. 5. Shahidi S, Zamiri B, Momeni Danaei S, Salehi S,
Hamedani S. Evaluation of anatomic variations in maxillary sinus with the aid of Cone Beam Computed Tomography (CBCT) in a Population in South of Iran. J Dent (Shiraz). 2016; 17(1):7-15.

6. 6. Gonzales $\mathrm{H}$, Peñarrocha $\mathrm{M}$, Garinos J, Sorní M. A study of the septa in the maxillary sinuses and the subantral alveolar processes in 30 patients. J Oral Implantol. 2007; 33(6):340-3.

7. 7. Rosano G, Taschierti S, Gaudy J, Lesmes D, Fabro M. Maxillary sinus septa: a cadaveric study. J Oral Maxillofac Surg. 2010; 68(6):1360-4.

8. 8. Lana JP, Carneiro PM, Machado Vde C, de Souza PE, Manzi FR, Horta MC. Anatomic variations and lesions of the maxillary sinus detected in cone beam computed tomography for dental implants. Clin Oral Implants Res. 2012; 23(12):1398-403.

9. 9. Uemura J. Morphological studies on the maxilla of the endentulous skulls and the skulls with teeth. On the sinus of the maxilla (author's transl). Shikwa Gakuho. 1974;74(12):1860-89.

10. 10. Ella B, Costa R, Lauverjat $Y$, et al. Septa within the sinus: effect on elevation of the sinus floor. $\mathrm{Br} \mathrm{J}$ Oral Maxillofac Surg. 2008; 46(6):464-7.

11. 11. Orhan K, Kusakci B, Aksoy S, Bayindir H, Berberoğlu A, Seker E. Cone beam CT evaluation of maxillary sinus septa prevalence, height, location and morphology in children and an adult population. Med Princ Pract. 2013;22(1):47-53.

12. 12. Özeç İ, Kiliç E, Müderris S. Maxillary sinus septa: evaluation with computed tomography and panoramic radiography. J Cumhuriyet Dent. 2008;11(2):82-6.

13. 13. Maestre-Ferrín L, Galán-Gil S, Rubio-Serrano M, Peñarrocha-Diago M, Peñarrocha-Oltra D. Maxillary sinus septa: a systematic review. Med Oral Patol Oral Cir Bucal. 2010; 15(2):e383-6.

14. 14. Underwwood A. An inquiry into the anatomy 
and pathology of the maxillary sinus. J Anat Physiol. 1910;44(4):354-69.

15. 15. Krennmair G, Ulm C, Lugmayr H. Maxillary sinus septa: incidence, morphology and clinical implications. J Cranio Maxillo Fac Surg. 1997; 25(5):261-5.

16. 16. Munetaka N, Yutaka S, Kenichi G, Masaki I, Shintaro K, Eiichiro A. Observation of maxillary sinus septa and bony bridges using dry skulls between Hellman's dental age of IA and IIIC. Okajimas Folia Anat Jpn. 2010;87(2):41-7.

17. 17. Ibrahim D, Burcu E, Şule K. Dental volumetric tomographical evaluation of location and prevalence of maxillary sinus septa. Cukurova Med J. 2013;38(3): 467-74.

18. 18. Van Zyl AW, V. A retrospective analysis of maxillary sinus septa on reformatted computerised tomography scans. Clin Oral Implants Res. 2009;20(12):1398401.

19. 19. Shen E, Fu E, Chiu T, Chang V, Chiang C, Tu H. Prevalence and location of maxillary sinus septa in the Taiwanese population and relationship to the absence of molars. Clin Oral Implants Res. 2012;23(6):741-5.

20. 20. Rancitelli D, Borgonovo $\mathrm{AE}$, Cicciù $\mathrm{M}$, et al. Maxillary sinus septa and anatomic correlation with the schneiderian membrane. J Craniofac Surg. 2015;26(4):1394-8.
21. 21. Echeverri S, Giraldo D, Lozano L, Mejía P, Montoya L, Vásquez E. Síndrome de visión por computador: una revisión de sus causas y del potencial de prevención. Rev CES Salud Pública. 2012;3(2):193-201.

22. 22. Neugebauer J, Ritter L, Mischkowski RA, et al. Evaluation of maxillary sinus anatomy by cone-beam CT prior to sinus floor elevation. Int J Oral Maxillofac Implants. 2010; 25(2): 258-65.

23. 23. Qian L, Tian XM, Zeng L, Gong Y, Wei B. Analysis of the morphology of maxillary sinus septa on Reconstructed Cone-Beam Computed Tomography Images. J Oral Maxillofac Surg. 2016;74(4):729-37.

24. 24. Cruz R. Características tomográficas de los septos del seno maxilar en pacientes de la clínica dental de la Universidad Peruana Cayetano Heredia (sede san Isidro), periodo 2015-2016. Tesis para obtener título de especialista. Lima: Universidad Peruana Cayetano Heredia; 2017.

Recibido: 24-10-2018

Aceptado: 05-01-2019 